\title{
Positive $\mu$-pseudo almost periodic solutions for some nonlinear infinite delay integral equations arising in epidemiology using Hilbert's projective metric
}

https://doi.org/10.1515/msds-2018-0007

Received July 5, 2017; accepted April 20, 2018

Abstract: The purpose of this work is to give sufficient conditions which guarantee the existence and the uniqueness of positive $\mu$-pseudo almost periodic solutions for the nonlinear infinite delay integral equation

$$
x(t)=\alpha(t) x(t-\beta)+\int_{-\infty}^{t} a(t, t-s) f(s, x(s)) \mathrm{d} s+h(t, x(t)) \quad \text { for } t \in \mathbb{R} .
$$

We improve the original work of [H. S. Ding, Y. Y. Chen and G. M. N'Guérékata, Existence of positive pseudo almost periodic solutions to a class of neutral integral equations, Nonlinear Analysis. Theorey, Methods and Applications 74 (2011) 7356-7364] by dropping the hypotheses of monotonicity on the functions $f$ and $h$. The main results are proved by using the Hilbert's projective metric combined with the contraction mapping principle. Our results can deal with some cases to which many results are not applicable. An example is provided to illustrate the main results of this work.

Keywords: $\mu$-pseudo almost periodic functions, Nonlinear delay integral equation, Contraction mapping principle, Hilbert's projective metric, Positive solution

MSC: 34C27, 45G10, 47H10

\section{Introduction}

In this work, we deal with the following nonlinear infinite delay integral equation

$$
x(t)=\alpha(t) x(t-\beta)+\int_{-\infty}^{t} a(t, t-s) f(s, x(s)) \mathrm{d} s+h(t, x(t)) \quad \text { for } t \in \mathbb{R},
$$

where $\beta$ is a constant and $\alpha, a, f$ and $h$ are continuous functions satisfying some hypotheses recalled in Section 3. This equation is more general than the following delay integral equation

$$
x(t)=\int_{t-\sigma}^{t} f(s, x(s)) \mathrm{d} s \quad \text { for } t \in \mathbb{R},
$$

\footnotetext{
Khalil Ezzinbi: Université Cadi Ayyad, Faculté des Sciences Semlalia, Département de Mathématiques, B.P. 2390, Marrakesh, Morocco, E-mail: ezzinbi@uca.ac.ma

^Corresponding Author: Mohamed Ziat: Université Cadi Ayyad, Faculté des Sciences Semlalia, Département de Mathématiques, B.P. 2390, Marrakesh, Morocco, E-mail: ziat.maths@gmail.com
} 
which can be considered as a model to explain the evolution of some infectious diseases: $x(t)$ is the proportion of infectious in population at time $t, \sigma$ is the length of time in which an individual remains infectious and $f(t, x(t))$ is the proportion of new infectious per unit of time.

Equation (1.2) has been studied in the periodic case in [1, 5, 6, 12-15, 21, 22] and in [11] in the almost periodic case. When the delay is state dependent, the existence of positive almost periodic solutions to the integral equation

$$
x(t)=\int_{t-\sigma(t)}^{t} f(s, x(s)) \mathrm{d} s \quad \text { for } t \in \mathbb{R}
$$

was considered in $[2,10,11,24]$. The approach used in $[10,11,24]$ is based in the Hilbert's projective metric.

Ding et al. studied in [20] the existence of positive almost automorphic solutions for the neutral nonlinear delay integral equation

$$
x(t)=\gamma x(t-\tau)+(1-\gamma) \int_{t-\sigma}^{t} f(s, x(s)) \mathrm{d} s \quad \text { for } t \in \mathbb{R},
$$

where $0 \leq \gamma<1, f(t, x)=\sum_{i=1}^{n} f_{i}(t, x) g_{i}(t, x), f_{i}(t, \cdot)$ is nondecreasing in $\mathbb{R}^{+}$and $g_{i}(t, \cdot)$ is nonincreasing in $\mathbb{R}^{+}$. Equation (1.4) is also studied in the almost periodic case by Ait Dads and Ezzinbi [4] where $f(t, \cdot)$ is nondecreasing in $\mathbb{R}^{+}$.

In 2011, Ding et al [19] considered the existence of positive solutions for Equation (1.1) where $f(t, x)=$ $\sum_{i=1}^{n} f_{i}(t, x) g_{i}(t, x), f_{i}(t, \cdot)$ is increasing in $\mathbb{R}^{+}, g_{i}(t, \cdot)$ is decreasing in $\mathbb{R}^{+}$and $h(t, \cdot)$ is decreasing in $\mathbb{R}^{+}$. They discussed the existence of positive pseudo almost periodic solutions by establishing a new fixed point theorem in partially ordered Banach spaces. The result of [19] cannot be applied when one of the function $f(t, \cdot)$ or $h(t, \cdot)$ is neither decreasing nor increasing in $\mathbb{R}^{+}$.

In the present work, we study the existence and the uniqueness of positive $\mu$-pseudo almost periodic solutions for Equation (1.1) without any hypothesis of monotonicity on the functions $f$ and $h$. Then we apply our result to a finite delay integral equation when the delay is state dependent; namely,

$$
x(t)=\alpha(t) x(t-\beta)+\int_{t-\sigma(t)}^{t} f(s, x(s)) \mathrm{d} s+h(t, x(t)) \quad \text { for } t \in \mathbb{R} .
$$

To obtain our results, we use the contraction mapping principle associated with the Hilbert's projective metric. Note that in the case when $\alpha \equiv 0$ and $h \equiv 0$, Equation (1.1) was considered using the Hilbert's projective metric by Ait Dads et al [3] in the pseudo almost periodic case and by Cieutat and Ezzinbi [17] in the pseudo almost automorphic case.

On the other hand, the notion of $\mu$-pseudo almost periodicity is more general than the one of pseudo almost periodicity [26-28]. It was introduced by Blot et al in [7] in order to correct many results about weighted pseudo almost periodicity $[8,9]$ like completeness and invariance by translation.

This work is organized as follows: In Section 2, we recall some preliminaries about the Hilbert's projective metric and $\mu$-pseudo almost periodic functions. In Section 3, we establish our main results on the existence and the uniqueness of $\mu$-pseudo almost periodic solutions for Equations (1.1) and (1.5). In Section 4, we illustrate our work by examining an example and we close by a remark which proves the advantage of this work.

\section{Preliminaries}

In this section, we recall some notions about $\mu$-pseudo almost periodic functions and the Hilbert's projective metric. For more details about theses topics, we refer the reader to [7] and [23]. 


\section{1 $\mu$-pseudo almost periodic functions}

In the sequel, we give some properties about $\mu$-pseudo almost periodic functions. Let $B C(\mathbb{R}, X)$ be the space of all bounded and continuous functions from $\mathbb{R}$ to a Banach space $X$, endowed with the uniform topology. Throughout this work, $X$ will be $\mathbb{R}$ the set of real numbers or $L^{1}\left(\mathbb{R}^{+}\right)$the Lebesgue space of order one in $\mathbb{R}^{+}$ endowed with the norm

$$
\|x\|_{L^{1}\left(\mathbb{R}^{+}\right)}=\int_{0}^{+\infty}|x(t)| \mathrm{d} t .
$$

Let $x \in B C(\mathbb{R}, X)$ and $\beta \in \mathbb{R}$. We define the function $\tau_{\beta} x$ by

$$
\left(\tau_{\beta} x\right)(s)=x(s-\beta) \quad \text { for } s \in \mathbb{R} .
$$

We denote by $\mathfrak{B}$ the Lebesgue $\sigma$-field of $\mathbb{R}$ and by $\mathcal{M}$ the set of all positive measures $\mu$ on $\mathfrak{B}$ satisfying $\mu(\mathbb{R})=+\infty$ and $\mu([a, b])<+\infty$, for all $a, b \in \mathbb{R}(a \leq b)$. From $\mu \in \mathcal{M}$, we formulate the following hypotheses taken from [7]:

(A) For all $a, b$ and $c \in \mathbb{R}$, such that $0 \leq a \leq b \leq c$, there exist $\tau_{0} \geq 0$ and $\alpha_{0}>0$ such that

$$
|\tau| \geq \tau_{0} \Rightarrow \mu((a+\tau, b+\tau)) \geq \alpha_{0} \mu([\tau, c+\tau]) .
$$

(B) For all $\tau \in \mathbb{R}$, there exist $\beta>0$ and a bounded interval $I$ such that

$$
\mu(\{a+\tau: a \in A\}) \leq \beta \mu(A) \text { when } A \in \mathfrak{B} \text { satisfies } A \cap I=\emptyset .
$$

In [7], the authors proved that

$$
\text { (B) } \Rightarrow(\mathbf{A}) \text {. }
$$

Definition 2.1. ([16]) A continuous function $f: \mathbb{R} \rightarrow X$ is said to be (in Bohr sense) almost periodic if for all $\varepsilon>0$, there exists $l>0$, such that for all $\alpha \in \mathbb{R}$, there exists $\tau \in[\alpha, \alpha+l]$ with

$$
\sup _{t \in \mathbb{R}}\|f(t+\tau)-f(t)\|<\varepsilon
$$

$A P(\mathbb{R}, X)$ denotes the space of the almost periodic $X$ valued functions.

Definition 2.2. ([25]) A continuous function $f: \mathbb{R} \times X \rightarrow Y$ is said to be almost periodic in $t$ uniformly with respect to $x \in X$ if for each compact set $K$ in $X$, for all $\varepsilon>0$, there exists $l>0$, such that for any $\alpha \in \mathbb{R}$, there exists $\tau \in[\alpha, \alpha+l]$ with

$$
\sup _{t \in \mathbb{R}} \sup _{x \in K}\|f(t+\tau, x)-f(t, x)\|<\varepsilon .
$$

Denote by $A P U(\mathbb{R} \times X, Y)$ the set of such functions.

Definition 2.3. ([7]) Let $\mu \in \mathcal{M}$. A bounded continuous function $f: \mathbb{R} \rightarrow X$ is said to be $\mu$-ergodic if

$$
\lim _{r \rightarrow+\infty} \frac{1}{\mu([-r, r])} \int_{[-r, r]}\|f(t)\| \mathrm{d} \mu(t)=0 .
$$

We denote the set of all such functions by $\varepsilon(\mathbb{R}, X, \mu)$.

Definition 2.4. ([7]) Let $\mu \in \mathcal{M}$. A continuous function $f: \mathbb{R} \rightarrow X$ is said to be $\mu$-pseudo almost periodic if $f$ is written in the form $f=g+\phi$ where $g \in A P(\mathbb{R}, X)$ and $\phi \in \mathcal{E}(\mathbb{R}, X, \mu)$.

We denote the space of all such functions by $P A P(\mathbb{R}, X, \mu)$. One can easily check that

$$
A P(\mathbb{R}, X) \subset P A P(\mathbb{R}, X, \mu) \subset B C(\mathbb{R}, X) .
$$


Example 2.5. ([7]) Let $\rho$ be a nonnegative $\mathfrak{B}$-measurable function. Denote by $\mu$ the positive measure defined by

$$
\mu(A)=\int_{A} \rho(t) \mathrm{d} t \text { for } A \in \mathfrak{B}
$$

where $\mathrm{d} t$ denotes the Lebesgue measure on $\mathbb{R}$. The function $\rho$ which occurs in (2.2) is called the RadonNikodym derivative of $\mu$ with respect to the Lebesgue measure on $\mathbb{R}$. In this case $\mu \in \mathcal{M}$ if and only if its Radon-Nikodym derivative $\rho$ is locally-integrable on $\mathbb{R}$ and it satisfies

$$
\int_{-\infty}^{+\infty} \rho(t) \mathrm{d} t=+\infty .
$$

Theorem 2.6. ([7]) Let $\mu \in \mathcal{M}$ satisfy (A). Then the decomposition of a $\mu$-pseudo almost periodic in the form $f=g+\varphi$, where $g \in A P(\mathbb{R}, X)$ and $\varphi \in \mathcal{E}(\mathbb{R}, X, \mu)$, is unique.

Remark 2.7. In what follows, if $\mu \in \mathcal{M}$ satisfies (A) and $f \in P A P(\mathbb{R}, X, \mu)$, then $f^{a p}$ and $f^{e r}$ denote respectively the almost periodic and the ergodic component of $f$.

Theorem 2.8. ([7]) Let $\mu \in \mathcal{M}$ satisfy (A). Then $\left(P A P(\mathbb{R}, X, \mu),\|\cdot\|_{\infty}\right)$ is a Banach space.

Theorem 2.9. ([7]) Let $\mu \in \mathcal{M}$ satisfy (B). Then $P A P(\mathbb{R}, X, \mu)$ is invariant by translation, that is $f \in$ $P A P(\mathbb{R}, X, \mu)$ implies $\tau_{\beta} f \in P A P(\mathbb{R}, X, \mu)$ for all $\beta \in \mathbb{R}$ where $\tau_{\beta} f$ is given by (2.1).

Definition 2.10. ([7]) Let $\mu \in \mathcal{M}$. A continuous function $f: \mathbb{R} \times X \rightarrow Y$ is said to be $\mu$-ergodic in $t$ uniformly with respect to $x \in X$ if the two following conditions are true:

i) for all $x \in X, f(\cdot, x) \in \mathcal{E}(\mathbb{R}, Y, \mu)$,

ii) $f$ is uniformly continuous on each compact set $K$ in $X$ with respect to the second variable $x$.

Denote by $\mathcal{E} U(\mathbb{R} \times X, Y, \mu)$ denotes the set of all such functions.

Definition 2.11. ([7]) Let $\mu \in \mathcal{M}$. A continuous function $f: \mathbb{R} \times X \rightarrow Y$ is said to be $\mu$-pseudo almost periodic in $t$ uniformly with respect to $x$ in $X$ if $f$ is written in the form: $f=g+\phi$, where $g \in A P U(\mathbb{R} \times X, Y)$ and $\phi \in \mathcal{E} U(\mathbb{R} \times X, Y, \mu)$.

$P A P U(\mathbb{R} \times X, Y, \mu)$ denotes the set of such functions. We have

$$
A P U(\mathbb{R} \times X, Y) \subset P A P U(\mathbb{R} \times X, Y, \mu) .
$$

Theorem 2.12. ([7]) Let $\mu \in \mathcal{M}, f \in P A P U(\mathbb{R} \times X, Y, \mu)$ and $x \in P A P(\mathbb{R}, X, \mu)$. Assume that the following hypothesis holds:

(C) For all bounded subset $B$ of $X$, $f$ is bounded on $\mathbb{R} \times B$.

Then $[t \mapsto f(t, x(t))] \in P A P(\mathbb{R}, Y, \mu)$.

\subsection{Hilbert's projective metric}

Let $\mathbb{X}$ be real Banach space. A closed convex set $K$ in $\mathbb{X}$ is called a convex cone if the following conditions are satisfied:

(i) if $x \in K$, then $\lambda x \in K$ for $\lambda \geq 0$

(ii) if $x \in K$ and $-x \in K$, then $x=0$.

A cone $K$ induces a partial ordering $\leq$ in $\mathbb{X}$ by

$$
0 \leq x \leq y \quad \text { if and only if } \quad y-x \in K .
$$

A cone $K$ is called normal if there exists a constant $k$ such that

$$
x \leq y \text { implies that }\|x\| \leq k\|y\|
$$


where $\|\cdot\|$ is the norm on $\mathbb{X}$. If $K$ is now a general cone in a Banach space $\mathbb{X}$ and $x$ and $y$ are elements of $K^{\star}=K-\{0\}$, we say that $x$ and $y$ are comparable if there exist real numbers $\alpha>0$ and $\beta>0$ such that

$$
\alpha x \leq y \leq \beta x .
$$

This define an quivalence relation on $K^{\star}$ and divides $K^{\star}$ into disjoint subsets which we call components of $K$. If $x$ and $y$ are comparable, we define the numbers $m(y / x)$ and $M(y / x)$ by

$$
\begin{aligned}
& m(y / x):=\sup \{\alpha>0 ; \alpha x \leq y\} \\
& M(y / x):=\inf \{\beta>0 ; y \leq \beta x\} .
\end{aligned}
$$

We define a metric which was introduced by Thompson [23]. If $x$ and $y \in K^{\star}$ are comparable, define $d(x, y)$ by

$$
\begin{aligned}
d(x, y) & :=\max (\log M(y / x), \log M(x / y)) \\
& =\max (\log M(y / x),-\log m(y / x)) .
\end{aligned}
$$

If $C$ is a component of $K$, one can easily prove [23] that $d$ gives a metric on $C$. Moreover Thompson proves the following result.

Theorem 2.13. ([23]) Let $K$ be a normal cone in a Banach space $\mathbb{X}$ and let $C$ be a component of $K$. Then $C$ is a complete metric space with respect to the metric $d$.

Proposition 2.14. ([23]) Let $K$ be a normal cone in a Banach space $\mathbb{X}$ with nonempty interior $\stackrel{\circ}{K}$. Then $\stackrel{\circ}{K}$ is a component of $K$.

It follows from Theorem (2.13) and Proposition (2.14) that if $K$ is a normal cone with nonempty interior $\stackrel{\circ}{K}$, then $\stackrel{\circ}{K}$ is a complete metric space with respect to the metric $d$.

\section{Main result}

In this section, we discuss the existence and the uniqueness of $\mu$-pseudo almost periodic solutions of Equations (1.1) and (1.5) with a positive infimum.

Equations (1.1) and (1.5) will be studied under the following Hypotheses.

(H1) $\alpha \in P A P(\mathbb{R}, \mathbb{R}, \mu)$ such that $\alpha(t) \geq 0$ for $t \in \mathbb{R}$ and $\sup _{t \in \mathbb{R}}|\alpha(t)|<1$.

(H2) $f$ and $h$ are nonnegative functions belonging to $P A P U(\mathbb{R} \times \mathbb{R}, \mathbb{R}, \mu)$.

(H3)There exist $\varphi$ and $\phi:(0,1) \times(0,+\infty) \rightarrow(0,1]$ and $k \in[0,1)$ such that $\varphi(\lambda, x)>\lambda^{k}$ and $\phi(\lambda, x)>\lambda^{k}$ and

$$
\lambda x \leq y \leq \lambda^{-1} x \Rightarrow\left\{\begin{array}{l}
f(t, y) \geq \varphi(\lambda, x) f(t, x) \\
h(t, y) \geq \phi(\lambda, x) h(t, x)
\end{array}\right.
$$

for each $x, y \in(0,+\infty), t \in \mathbb{R}$ and $\lambda \in(0,1)$.

(H4) $a: \mathbb{R} \times \mathbb{R}^{+} \rightarrow \mathbb{R}^{+}$such that the function $t \mapsto a(t, \cdot)$ is in $\operatorname{PAP}\left(\mathbb{R}, L^{1}\left(\mathbb{R}^{+}\right), \mu\right)$. Moreover, there exists $b \in L^{1}\left(\mathbb{R}^{+}\right)$such that $\left|a^{a p}(t, s)\right| \leq b(s)$ for all $t \in \mathbb{R}$ and almost every where $s \in \mathbb{R}^{+}$, where $t \mapsto a^{a p}(t, \cdot)$ is the almost periodic component of $t \mapsto a(t, \cdot)$.

(H5)There exists $x_{0}>0$ such that

$$
\inf _{t \in \mathbb{R}} \int_{0}^{+\infty} a(t, s) f\left(t-s, x_{0}\right) \mathrm{d} s>0 .
$$


To give the proof of the main results, we need the following three lemmas.

Lemma 3.1. Let $\mu \in \mathcal{M}$ satisfy (B) and $a: \mathbb{R} \times \mathbb{R}^{+} \rightarrow \mathbb{R}^{+}$such that the function $t \mapsto a(t, \cdot)$ is in $\operatorname{PAP}\left(\mathbb{R}, L^{1}\left(\mathbb{R}^{+}\right), \mu\right)$. We assume that there exists $b \in L^{1}\left(\mathbb{R}^{+}\right)$such that $\left|a^{a p}(t, s)\right| \leq b(s)$ for all $t \in \mathbb{R}$ and almost every where $s \in \mathbb{R}^{+}$. If $f \in P A P(\mathbb{R}, \mathbb{R}, \mu)$ then the function $F$ defined by

$$
F(t)=\int_{-\infty}^{t} a(t, t-s) f(s) \mathrm{d} s
$$

is also $\mu$-pseudo almost periodic. Furthermore, the almost periodic component of $F$ is given by

$$
F^{a p}(t)=\int_{-\infty}^{t} a^{a p}(t, t-s) f^{a p}(s) \mathrm{d} s
$$

Proof. To prove that $F$ is $\mu$-pseudo almost periodic and the almost periodic component is given by (3.2), we must prove that $\lim _{r \rightarrow+\infty} A(r)=0$ where

$$
A(r)=\frac{1}{\mu([-r, r])} \int_{[-r, r]}\left|\int_{-\infty}^{t}\left(a(t, t-s) f(s)-a^{a p}(t, t-s) f^{a p}(s)\right) \mathrm{d} s\right| \mathrm{d} \mu(t) .
$$

From $\mu(\mathbb{R})=+\infty$, it follows $\mu([-r, r])>0$ for $r$ sufficiently large. Since

$$
A(r)=\frac{1}{\mu([-r, r])} \int_{[-r, r]}\left|\int_{-\infty}^{t}\left(a^{e}(t, t-s) f(s)+a^{a p}(t, t-s) f^{e}(s)\right) \mathrm{d} s\right| \mathrm{d} \mu(t),
$$

we obtain

$$
\begin{aligned}
A(r) \leq & \|f\|_{\infty} \frac{1}{\mu([-r, r])} \int_{[-r, r]}\left\|a^{e}(t, \cdot)\right\|_{L^{1}\left(\mathbb{R}^{+}\right)} \mathrm{d} \mu(t) \\
& +\frac{1}{\mu([-r, r])} \int_{[-r, r]}\left(\int_{0}^{+\infty}\left|a^{a p}(t, s) f^{e}(t-s)\right| \mathrm{d} s\right) \mathrm{d} \mu(t) .
\end{aligned}
$$

By the Hypothesis, one has

$$
\lim _{r \rightarrow+\infty} \frac{1}{\mu([-r, r])} \int_{[-r, r]}\left\|a^{e}(t, \cdot)\right\|_{L^{1}\left(\mathbb{R}^{+}\right)} \mathrm{d} \mu(t)=0 .
$$

Using the Fubini Theorem, we get

$$
\frac{1}{\mu([-r, r])} \int_{[-r, r]}\left(\int_{0}^{+\infty}\left|a^{a p}(t, s) f^{e}(t-s)\right| \mathrm{d} s\right) \mathrm{d} \mu(t) \leq \int_{0}^{+\infty} b(s)\left(\frac{1}{\mu([-r, r])} \int_{[-r, r]}\left|f^{e}(t-s)\right| \mathrm{d} \mu(t)\right) \mathrm{d} s .
$$

Since $\mu$ satisfies (B), then from Theorem 2.9, we have $\left[t \mapsto f^{e}(t-s)\right] \in \mathcal{E}(\mathbb{R}, \mathbb{R}, \mu)$ for every $s \in \mathbb{R}$. Therefore

$$
\begin{gathered}
\lim _{r \rightarrow+\infty} \frac{1}{\mu([-r, r])} \int_{[-r, r]}\left|f^{e}(t-s)\right| \mathrm{d} \mu(t)=0, \\
\left|b(s)\left(\frac{1}{\mu([-r, r])} \int_{[-r, r]}\left|f^{e}(t-s)\right| \mathrm{d} \mu(t)\right)\right| \leq\left\|f^{e}\right\|_{\infty} b(s) \quad \text { for } s \in[0,+\infty) .
\end{gathered}
$$

Using the Lebesgue Dominated Convergence Theorem, we deduce that $\lim _{r \rightarrow+\infty} A(r)=0$. This ends the proof of Lemma 3.1. 
Lemma 3.2. Let $\mu \in \mathcal{M}$. Assume that $f \in P A P(\mathbb{R}, \mathbb{R}, \mu)$ and $g \in P A P(\mathbb{R}, \mathbb{R}, \mu)$. Then we have $f g \in$ $\operatorname{PAP}(\mathbb{R}, \mathbb{R}, \mu)$.

Proof. Since one has $f g=f^{a p} g^{a p}+f^{a p} g^{e}+f^{e} g$, it suffices to show that $f^{a p} g^{e}+f^{e} g$ is $\mu$ ergodic. Set

$$
I(r):=\frac{1}{\mu([-r, r])} \int_{[-r, r]}\left|\left(f^{a p} g^{e}+f^{e} g\right)(t)\right| \mathrm{d} \mu(t) .
$$

We must prove that $\lim _{r \rightarrow+\infty} I(r)=0$. For $r$ sufficiently large we have

$$
I(r) \leq \frac{\left\|f^{a p}\right\|_{\infty}}{\mu([-r, r])} \int_{[-r, r]}\left|g^{e}(t)\right| \mathrm{d} \mu(t)+\frac{\|g\|_{\infty}}{\mu([-r, r])} \int_{[-r, r]}\left|f^{e}(t)\right| \mathrm{d} \mu(t) .
$$

By the hypothesis, one has

$$
\frac{1}{\mu([-r, r])} \int_{[-r, r]}\left|g^{e}(t)\right| \mathrm{d} \mu(t) \rightarrow 0 \quad \text { as } \quad r \rightarrow+\infty,
$$

and

$$
\frac{1}{\mu([-r, r])} \int_{[-r, r]}\left|f^{e}(t)\right| \mathrm{d} \mu(t) \rightarrow 0 \quad \text { as } \quad r \rightarrow+\infty .
$$

Then by (3.3)-(3.5), we obtain that $\lim _{r \rightarrow+\infty} I(r)=0$. This ends the proof of Lemma 3.2.

Lemma 3.3. Suppose that the following hypotheses hold:

(h1) $f \in C\left(\mathbb{R} \times \mathbb{R}^{+}, \mathbb{R}^{+}\right)$and there exists $x_{1}>0$ such that $f\left(\cdot, x_{1}\right) \in B C(\mathbb{R}, \mathbb{R})$.

(h2)There exists a map $\xi:(0,1) \times(0,+\infty) \rightarrow[0,1)$ satisfying $\xi(\lambda, x)>\lambda^{k}$ and

$$
\lambda x \leq y \leq \lambda^{-1} x \Rightarrow f(t, y) \geq \xi(\lambda, x) f(t, x)
$$

for each $x, y>0, t \in \mathbb{R}$ and $\lambda \in(0,1)$.

Then we have

i) $f(t, y) \geq\left(\min \left(\frac{x}{y}, \frac{y}{x}\right)\right)^{k} f(t, x)$ for $x, y>0$ and $t \in \mathbb{R}$.
ii) For each $[a, b] \subset(0,+\infty)$, $f$ is bounded on $\mathbb{R} \times[a, b]$.

Proof. i) Let $x, y>0$ and $t \in \mathbb{R}$. If $x=y$ then $f(t, y)=\left(\min \left(\frac{x}{y}, \frac{y}{x}\right)\right)^{k} f(t, x)$. Suppose that $x \neq y$ and take $\lambda=\min \left(\frac{x}{y}, \frac{y}{x}\right)$. Then $\lambda x \leq y \leq \lambda^{-1} x$. Hypothesis (h2) gives us

$$
f(t, y) \geq \xi(\lambda, x) f(t, x)>\lambda^{k} f(t, x)=\left(\min \left(\frac{x}{y}, \frac{y}{x}\right)\right)^{k} f(t, x) .
$$

ii) Let $x \in[a, b]$. By i), it follows that

$$
f\left(t, x_{1}\right) \geq\left(\min \left(\frac{x}{x_{1}}, \frac{x_{1}}{x}\right)\right)^{k} f(t, x) \geq\left(\min \left(\frac{a}{x_{1}}, \frac{x_{1}}{b}\right)\right)^{k} f(t, x) .
$$

The desired result follows from Hypothesis (h1). The proof of Lemma 3.3 is now complete.

Now, we give the first result of this work which shows the existence and the uniqueness of positive $\mu$-pseudo almost periodic solutions for Equation (1.1). 
Theorem 3.4. Let $\mu \in \mathcal{N}$ satisfy (B). Suppose that (H1)-(H5) hold. Then Equation (1.1) has a unique $\mu$-pseudo almost periodic solution $x^{\star}$ with a positive infimum.

Proof. Let $\mathbb{X}=P A P(\mathbb{R}, \mathbb{R}, \mu)$ be the space of $\mu$-pseudo almost periodic functions endowed with the norm defined by $\|f\|_{\infty}=\sup _{t \in \mathbb{R}}|f(t)|$. Since $\mu \in \mathcal{N}$ satisfies (B), then By Theorem 2.8, $\mathbb{X}$ is a Banach space. Let $K$ be the cone of nonnegative functions in $P A P(\mathbb{R}, \mathbb{R}, \mu)$. Then $K$ is a normal convex cone. Furthermore, we have

$$
0 \leq x \leq y \quad \Rightarrow \quad\|x\|_{\infty} \leq\|y\|_{\infty} .
$$

The interior of $K$ is given by $\stackrel{\circ}{K}=\left\{x \in P A P(\mathbb{R}, \mathbb{R}, \mu): \inf _{t \in \mathbb{R}} x(t)>0\right\}$. Define the operator $A$ on $B C(\mathbb{R}, \mathbb{R})$ by

$$
(A x)(t)=\alpha(t) x(t-\beta)=\alpha(t) \tau_{\beta} x(t) \quad \text { for } t \in \mathbb{R} .
$$

From (H1), $A$ is bounded linear operator from $B C(\mathbb{R}, \mathbb{R})$ to $B C(\mathbb{R}, \mathbb{R})$ and

$$
\|A\| \leq \sup _{t \in \mathbb{R}}|\alpha(t)|<1 .
$$

It follows that $(I-A)^{-1}$ exists and $B=(I-A)^{-1}=\sum_{k=0}^{+\infty} A^{k}$. Furthermore

$$
B=I+\sum_{n \geq 1}\left(\prod_{k=0}^{n-1} \tau_{k \beta} \alpha\right) \tau_{n \beta} .
$$

If we set $u(t)=x(t)-\alpha(t) x(t-\beta)=((I-A) x)(t)$, then Equation (1.1) is equivalent to the following equation

$$
u(t)=\int_{-\infty}^{t} a(t, t-s) f(s,(B u)(s)) \mathrm{d} s+h(t,(B u)(t)) .
$$

Since $\mu$ satisfies $(\mathbf{B})$, it follows from Theorem 2.9 that $P A P(\mathbb{R}, \mathbb{R}, \mu)$ is invariant by translation. In addition, since $\alpha \in P A P(\mathbb{R}, \mathbb{R}, \mu)$, Lemma 3.2 yields that

$$
B(P A P(\mathbb{R}, \mathbb{R}, \mu)) \subset P A P(\mathbb{R}, \mathbb{R}, \mu) .
$$

From (H3) and ii) of Lemma 3.3 we deduce that functions $f$ and $h$ satisfy condition (C) of Theorem 2.12. Using (H2), Theorem 2.12 and Lemma 3.3 we obtain

$$
u \in P A P(\mathbb{R}, \mathbb{R}, \mu) \Rightarrow[t \mapsto f(t,(B u)(t))] \in P A P(\mathbb{R}, \mathbb{R}, \mu) \quad \text { and } \quad[t \mapsto h(t,(B u)(t))] \in P A P(\mathbb{R}, \mathbb{R}, \mu) .
$$

On the other hand, in view of Hypothesis (H4) and Lemma 3.1, we get that

$$
u \in P A P(\mathbb{R}, \mathbb{R}, \mu) \Rightarrow\left[t \mapsto \int_{-\infty}^{t} a(t, t-s) f(s,(B u)(s)) \mathrm{d} s\right] \in P A P(\mathbb{R}, \mathbb{R}, \mu) .
$$

Now we have proved that

$$
u \in P A P(\mathbb{R}, \mathbb{R}, \mu) \Rightarrow\left[t \mapsto \int_{-\infty}^{t} a(t, t-s) f(s,(B u)(s)) \mathrm{d} s+h(t,(B u)(t))\right] \in P A P(\mathbb{R}, \mathbb{R}, \mu) .
$$

We claim that $x \in \stackrel{\circ}{K}$ is a solution of Equation (1.1) if and only if $u \in \stackrel{\circ}{K}$ is a solution of Equation (3.8).

Let $x \in \stackrel{\circ}{K}$ be a solution of Equation (1.1). Then there exists $\varepsilon>0$ such that

$$
\varepsilon<x(t)<\varepsilon^{-1} \text { for } t \in \mathbb{R} .
$$


In view of (H3) and Lemma 3.3, it follows that

$$
\begin{aligned}
u(t) & =x(t)-\alpha(t) x(t-\beta) \\
& =\int_{-\infty}^{t} a(t, t-s) f(s, x(s)) \mathrm{d} s+h(t, x(t)) \\
& \geq \int_{-\infty}^{t} a(t, t-s) f(s, x(s)) \mathrm{d} s \\
& \geq \int_{-\infty}^{t} a(t, t-s)\left(\min \left(\frac{x_{0}}{x(s)}, \frac{x(s)}{x_{0}}\right)\right)^{k} f\left(s, x_{0}\right) \mathrm{d} s \\
& \geq \varepsilon^{k}\left(\min \left(x_{0}, \frac{1}{x_{0}}\right)\right)^{k} \int_{-\infty}^{t} a(t, t-s) f\left(s, x_{0}\right) \mathrm{d} s \\
& \geq \varepsilon^{k}\left(\min \left(x_{0}, \frac{1}{x_{0}}\right)\right)^{k} \inf _{t \in \mathbb{R}} \int_{0}^{+\infty} a(t, s) f\left(t-s, x_{0}\right) \mathrm{d} s .
\end{aligned}
$$

Taking into account (H5), we get that $\inf _{t \in \mathbb{R}} u(t)>0$. On the other hand, since $x$ and $\alpha$ are $\mu$-pseudo almost periodic, $u$ is also $\mu$-pseudo almost periodic. Hence $u \in \stackrel{\circ}{K}$. Moreover $u$ is a solution of Equation (3.8).

Inversely, suppose that $u \in \stackrel{\circ}{K}$ is a solution of Equation (3.8) and let $t \in \mathbb{R}$. By (3.7), we have $B(u)(t) \geq u(t)$. Since $x(t)=(B u)(t)$ we obtain that $x(t) \geq u(t)$. Then $\inf _{t \in \mathbb{R}} x(t) \geq \inf _{t \in \mathbb{R}} u(t)>0$. Using (3.9), it follows that $x$ is $\mu$-pseudo almost periodic and consequently $x \in \stackrel{\circ}{K}$. However $x$ is a solution of Equation (1.1).

We denote by $T$ the operator associated with the right-hand side of Equation (3.8). Namely,

$$
(T x)(t)=\int_{-\infty}^{t} a(t, t-s) f(s,(B x)(s)) \mathrm{d} s+h(t,(B x)(t)) .
$$

Next, we prove that the opertor $T$ is a contraction. We consider

$$
\left(T_{1} x\right)(t)=\int_{-\infty}^{t} a(t, t-s) f(s,(B x)(s)) \mathrm{d} s \quad \text { and } \quad\left(T_{2} x\right)(t)=h(t,(B x)(t)) .
$$

From the discussion above (see (3.10)), the operator $T$ maps $P A P(\mathbb{R}, \mathbb{R}, \mu)$ into itself. Let $x \in \stackrel{\circ}{K}$. Then from (3.7) and (3.9), $B x \in \stackrel{\circ}{K}$. Hence, there exists $\varepsilon>0$ such that

$$
\varepsilon<(B x)(t)<\varepsilon^{-1} \text { for } t \in \mathbb{R} .
$$

In view of Lemma 3.3, we obtain

$$
\begin{aligned}
(T x)(t) & \geq \int_{-\infty}^{t} a(t, t-s) f(s,(B x)(s)) \mathrm{d} s \\
& \geq \int_{-\infty}^{t} a(t, t-s)\left(\min \left(\frac{x_{0}}{(B x)(s)}, \frac{(B x)(s)}{x_{0}}\right)\right)^{k} f\left(s, x_{0}\right) \mathrm{d} s \\
& \geq \varepsilon^{k}\left(\min \left(x_{0}, \frac{1}{x_{0}}\right)\right)^{k} \int_{-\infty}^{t} a(t, t-s) f\left(s, x_{0}\right) \mathrm{d} s
\end{aligned}
$$




$$
\geq \varepsilon^{k}\left(\min \left(x_{0}, \frac{1}{x_{0}}\right)\right)^{k} \inf _{t \in \mathbb{R}} \int_{0}^{+\infty} a(t, s) f\left(t-s, x_{0}\right) \mathrm{d} s .
$$

Therefore, by (H5), we obtain that $\inf _{t \in \mathbb{R}}(T x)(t)>0$ and consequently $T x \in \stackrel{\circ}{K}$ for all $x \in \stackrel{\circ}{K}$. So, $T$ maps $\stackrel{\circ}{K}$ into itself.

Let $x, y \in \stackrel{\circ}{K}$ and $\lambda \in(0,1)$ such that $\lambda x \leq y \leq \lambda^{-1} x$. It follows from (H3) that

$$
\left\{\begin{array}{l}
f(t, y(t)) \geq \varphi(\lambda, x(t)) f(t, x(t)) \\
h(t, y(t)) \geq \phi(\lambda, x(t)) h(t, x(t))
\end{array}\right.
$$

for $t \in \mathbb{R}$. We have also $\lambda y \leq x \leq \lambda^{-1} y$. Then

$$
\left\{\begin{array}{l}
\varphi(\lambda, x(t)) f(t, x(t)) \leq f(t, y(t)) \leq(\varphi(\lambda, x(t)))^{-1} f(t, x(t)) \\
\phi(\lambda, x(t)) h(t, x(t)) \leq h(t, y(t)) \leq(\phi(\lambda, x(t)))^{-1} h(t, x(t))
\end{array}\right.
$$

for $t \in \mathbb{R}$. This gives us

$$
\left\{\begin{array}{l}
\lambda^{k} f(t, x(t)) \leq f(t, y(t)) \leq \lambda^{-k} f(t, x(t)) \\
\lambda^{k} h(t, x(t)) \leq h(t, y(t)) \leq \lambda^{-k} h(t, x(t))
\end{array}\right.
$$

for $t \in \mathbb{R}$. Multiplying the first inequality of (3.12) by $a(t, t-s)$ and integrating over $(-\infty, t)$ we obtain for $t \in \mathbb{R}$

$$
\lambda^{k}\left(T_{1} x\right)(t) \leq\left(T_{1} y\right)(t) \leq \lambda^{-k}\left(T_{1} x\right)(t)
$$

and

$$
\lambda^{k}\left(T_{2} x\right)(t) \leq\left(T_{2} y\right)(t) \leq \lambda^{-k}\left(T_{2} x\right)(t)
$$

Summing (3.13) and (3.14) we obtain that

$$
\lambda^{k} T x \leq T y \leq \lambda^{-k} T x
$$

For $\lambda=\left(\max \left(M\left(\frac{y}{x}\right), M\left(\frac{x}{y}\right)\right)\right)^{-1}$, we deduce that $d(x, y)=\ln \left(\lambda^{-1}\right)$. Using (2.3)-(2.5), inequality (3.15) leads to

$$
d(T x, T y) \leq \ln \left(\lambda^{-k}\right)
$$

This shows that

$$
d(T x, T y) \leq k \ln \left(\lambda^{-1}\right)=k d(x, y) .
$$

We know from Theorem 2.13 and Proposition 2.14 that $\stackrel{\circ}{K}, d)$ is a complete metric space. In view of the contraction mapping principle, the operator $T$ has a unique fixed point $x^{\star} \in \stackrel{\circ}{K}$ which means that Equation (1.1) has a unique $\mu$-pseudo almost periodic solution with a positive infimum. The proof of Theorem 3.4 is now complete.

Next, we give the second result of this work which is a corollary of Theorem 3.4 about Equation (1.5).

Corollary 3.5. Let $\mu \in \mathcal{M}$ satisfy (B). Suppose that (H1)-(H3) hold. In addition, we assume that

i) $\sigma$ is a positive $\mu$-pseudo almost periodic function.

ii) There exists $x_{0}>0$ such that

$$
\inf _{t \in \mathbb{R}} \int_{t-\sigma(t)}^{t} f\left(s, x_{0}\right) \mathrm{d} s>0
$$

Then Equation (1.5) has a unique $\mu$-pseudo almost periodic solution $x^{\star}$ with a positive infimum. 
Proof. Equation (1.5) can be transformed into Equation (1.1) by letting

$$
a(t, s)=\mathbf{1}_{[0, \sigma(t)]}(s)= \begin{cases}1, & s \in[0, \sigma(t)] \\ 0, & s \notin[0, \sigma(t)]\end{cases}
$$

Obviously (3.17) implies (H5). Now let us prove that (H4) holds. By using the fact that

$$
\begin{cases} & \left\|\mathbf{1}_{\left[0, \sigma^{a p}(t+\tau)\right]}-\mathbf{1}_{\left[0, \sigma^{a p}(t)\right]}\right\|_{L^{1}\left(\mathbb{R}^{+}\right)}=\left|\sigma^{a p}(t+\tau)-\sigma^{a p}(t)\right| \\ & \left\|\mathbf{1}_{[0, \sigma(t)]}-\mathbf{1}_{\left[0, \sigma^{a p}(t)\right]}\right\|_{L^{1}\left(\mathbb{R}^{+}\right)}=\left|\sigma(t)-\sigma^{a p}(t)\right|\end{cases}
$$

we deduce from i) that $t \mapsto a(t, \cdot)$ is in $\operatorname{PAP}\left(\mathbb{R}, L^{1}\left(\mathbb{R}^{+}\right), \mu\right)$ with $a^{a p}(t, \cdot)=\mathbf{1}_{\left[0, \sigma^{a p}(t)\right]}(\cdot)$. Furthermore, we have $\left|a^{a p}(t, s)\right| \leq \mathbf{1}_{\left[0,\left\|\sigma^{a p}\right\|_{\infty}\right]}(s)$ and consequently (H4) is satisfied. By applying Theorem 3.4, we obtain the desired result.

\section{Application}

In this section, we present an example to illustrate the theory of this work. We also give a remark proving the advantage of the main results.

Example 4.1. We consider the measure $\mu$ where its Radon-Nikodym derivative is

$$
\rho(t)= \begin{cases}e^{t} & \text { for } t \leq 0 \\ 1 & \text { for } t>0\end{cases}
$$

It is well known from [7, Example 3.6, page 17] that $\mu \in \mathcal{M}$ satisfies (B). Let $\alpha(t) \equiv \frac{1}{5}, \beta=1$ and

$$
\begin{gathered}
a(t, s)=\frac{3-\sin \sqrt{2} t-\sin \pi t}{5\left(1+s^{2}\right)} \text { for } t \in \mathbb{R} \text { and } s \in \mathbb{R}^{+}, \\
f(t, x)=\left(1+\cos ^{2} t+\cos ^{2}(\pi t)+\frac{\pi}{2}-\arctan (t)\right) \sqrt[4]{\sqrt{|x|} \ln (1+\sqrt{|x|})}, \\
h(t, x)=\left(3+\sin (\pi t)+\sin (\sqrt{3} t)+\frac{1}{1+t^{2}}\right) \sqrt[4]{x^{2}+|x|}
\end{gathered}
$$

for $t$ and $x \in \mathbb{R}$. It is clear that the Hypothesis (H1) is satisfied and the functions $f$ and $h$ are nonnegative. On the other hand, $f$ and $h$ are $\mu$-pseudo almost periodic with $f^{e r}(t, x)=\left(\frac{\pi}{2}-\arctan (x)\right) \sqrt[4]{\sqrt{|x|} \ln (1+\sqrt{|x|})}$ and $h^{e r}(t, x)=\frac{\sqrt[4]{x^{2}+|x|}}{1+t^{2}}$. In fact, we have

$$
\begin{gathered}
\frac{1}{\mu([-r, r])} \int_{0}^{r}\left|\frac{\pi}{2}-\arctan (t)\right| \mathrm{d} t=\frac{\frac{\pi}{2} r+\ln \left(\sqrt{r^{2}+1}\right)-r \arctan (r)}{1-e^{-r}+r} \rightarrow 0 \text { as } r \rightarrow+\infty, \\
\frac{1}{\mu([-r, r])} \int_{-r}^{0}\left|\frac{\pi}{2}-\arctan (t)\right| e^{t} \mathrm{~d} t \leq \frac{1}{\mu([-r, r])} \int_{-r}^{0} \pi e^{t} \mathrm{~d} t \rightarrow 0 \text { as } r \rightarrow+\infty .
\end{gathered}
$$

For $h^{e r}$ we use [7, Corollary 2.15, page 8]. Consequently, Hypothesis (H2) holds. Define the functions

$$
\varphi(\lambda, x)=\lambda^{\frac{1}{8}} \sqrt[4]{\frac{\ln (1+\sqrt{\lambda x})}{\ln (1+\sqrt{x})}}, \quad \phi(\lambda, x)=\sqrt[4]{\frac{\lambda^{2} x^{2}+\lambda x}{x^{2}+x}} \quad \text { for } x>0 \text { and } \lambda \in(0,1)
$$

Then, we can easily prove that (3.1) is satisfied. Therefore, we have

$$
\varphi(\lambda, x)>\lambda^{\frac{1}{4}}>\lambda^{\frac{1}{2}}, \quad \phi(\lambda, x)>\lambda^{\frac{1}{2}} \quad \text { for } x>0 \text { and } \lambda \in(0,1) .
$$


Hypothesis $(\mathbf{H} 3)$ is now fulfilled with $k=\frac{1}{2}$. It is easy to show that Hypothesis $(\mathbf{H 4})$ holds with $b(s)=\left(1+s^{2}\right)^{-1}$ for $s \in \mathbb{R}^{+}$.

On the other hand, for $x>0$ and $y>0$ we have that

$$
\inf _{t \in \mathbb{R}} \int_{0}^{+\infty} a(t, s) f(t-s, x) \mathrm{d} s \geq \sqrt[4]{\sqrt{x} \ln (1+\sqrt{x})} \inf _{t \in \mathbb{R}} \int_{0}^{+\infty} \frac{\mathrm{d} s}{5\left(1+s^{2}\right)}=\frac{\pi}{10} \sqrt[4]{\sqrt{x} \ln (1+\sqrt{x})}>0,
$$

which yields that Hypothesis (H5) is satisfied. All the assumptions of Theorem 3.4 are verified. Hence, Equation (1.1) with the above functions $\alpha, a, f$ and $h$ has a unique $\mu$-pseudo almost periodic solution $x^{\star}$ with a positive infimum.

We close this work by the following interesting Remark.

Remark 4.2. (In the case $\mu$ is the Lebesgue measure)

i) Since for each $t \in \mathbb{R}$, the function $x \mapsto h(t, x)$ is not decreasing in $\mathbb{R}^{+}$, the result [19, Theorem 3.3] is not applicable to Example 4.1. This proves that this work is different from [19] and our consideration is significant. So, the advantage of this work is that no hypothesis of monotonicity is imposed on the functions $f$ and $h$.

ii) Let $p: \mathbb{R} \rightarrow \mathbb{R}$ be a nonnegative function such that $p$ is $\mu$-pseudo almost periodic. Then the function defined by the following

$$
h(t, x)= \begin{cases}p(t) \sqrt[3]{|x|} & \text { for } x \in[-1,1] \\ \frac{p(t)}{\sqrt[3]{|x|}} & \text { for } x \in(-\infty,-1] \cup[1,+\infty),\end{cases}
$$

is nonnegative and belongs to $\operatorname{PAP}(\mathbb{R} \times \mathbb{R}, \mathbb{R}, \mu)$. Define the function $\phi$ on $(0,1) \times(0,+\infty)$ by $\phi(\lambda, x)=\sqrt[3]{\lambda}$. Then for $x, y>0$ and $\lambda \in(0,1)$ we have

$$
\phi(\lambda, x)>\lambda^{\frac{1}{2}} \quad \text { and } \quad \lambda x \leq y \leq \lambda^{-1} x \quad \Rightarrow \quad h(t, y) \geq \phi(\lambda, x) h(t, x) .
$$

So, if we define the function $h$ in Example 4.1 by (4.3), we obtain the same conclusion. Also, [19, Theorem 3.3] cannot be applied to Example 4.1 because the function $x \mapsto h(t, x)$ is neither decreasing nor increasing in $\mathbb{R}^{+}$.

Acknowledgement: The authors are grateful to the referee for the valuable comments and suggestions which help us to improve the original manuscript.

\section{References}

[1] E. Ait Dads, O. Arino, K. Ezzinbi, Existence of periodic solution for some neutral nonlinear integral equation with delay time dependent. Facta Univ. Ser. Math. Inform. 11 (1996), 79-92.

[2] E. Ait Dads, O. Arino, K. Ezzinbi, Positive almost periodic solution for some nonlinear delay integral equation. Nonlinear Stud. 3 (1996), 85-101.

[3] E. Ait Dads, P. Cieutat, L. Lhachimi, Positive pseudo almost periodic solutions for some nonlinear infinite delay integral equations. Mathematical and Computer Modelling. 49 (2008), 721-739.

[4] E. Ait Dads, K. Ezzinbi, Almost periodic solutions for some neutral nonlinear integral equation. Nonlinear Anal. 28 (1997), 1479-1489.

[5] T.A. Burton, L. Hatvani, On the existence of periodic solutions of some nonlinear functional-differential equations with unbounded delay. Nonlinear Anal. 16 (1991), 389-396.

[6] s. Busenberg, K. Cooke, Periodic solutions to delay differential equations arising in some models of epidemiology. Applied Nonlinear Analysis. 67-78. Academic Press (1979).

[7] J. Blot, P. Cieutat, K. Ezzinbi, New approach for weightad pseudo almost functions under the light of measure theory, basic results and applications. Appl. Anal. (2011), 1-34. 
[8] T. Diagana, Weighted pseudo almost periodic functions and applications. C. R. Math. Acad. Sci. Paris. 343 (2006), 643-646.

[9] T. Diagana, Weighted pseudo almost periodic solutions to some differential equations. Nonlinear Anal. 68 (2008), 22502260.

[10] K. Ezzinbi, M. Hachimi, Existence of positive almost periodic solutions of functional equations via Hilbert's projective metric. Nonlinear Anal. 26 (1996), 1169-1176.

[11] A. Fink, J. Gatica, Positive almost periodic solutions of some delay integral equations. J. Differential Equations. 83 (1990), 166-178.

[12] D. Guo, V. Lakshmikantham, Positive solutions of nonlinear integral equations arising in infectious disease. J. Math. Anal. Appl. 134 (1988), 1-8.

[13] J. Kaplan, M. Sorg, J. Yorke, Solutions of $x^{\prime}(t)=f(x(t), x(t-L))$ have limits when $f$ is an order relation. Nonlinear Anal. 3 (1979), 53-58.

[14] R.W. Leggett, L.R. Williams, A fixed point theorem with application to an infectious disease model. J. Math. Anal. Appl. 76 (1980), 91-97.

[15] R.W. Leggett, L.R. Williams, Nonzero solutions of nonlinear integral equations modeling infectious disease. SIAM J. Math. Anal. 13 (1982), 112-121.

[16] C. Corduneanu, Almost Periodic Functions. Wiley, New York, 1968 (Reprented, Chelsea, New York, 1989).

[17] P. Cieutat, K. Ezzinbi, Positive pseudo almost automorphic solutions for some nonlinear infinite delay integral equations. Afr. Diaspora J. Math. 12 (2011), 19-33.

[18] K.L. Cooke, J.L. Kaplan, A periodic treshold theorem for epidemics and population growth. Math. Biosci. 3 (1976), 87-104.

[19] H. S. Ding, Y. Y. Chen, G. M. N'Guérékata, Existence of positive pseudo almost periodic solutions to a class of neutral integral equations. Nonlinear Anal. 74 (2011), 7356-7364.

[20] H. S. Ding, J. Liang, G. M. N'Guérékata, T. J. Xiao, Existence of positive almost automorphic solutions to neutral nonlinear integral equations. Nonlinear Anal. 69 (2008), 1188-1199.

[21] R. Nussbanum, A periodicity threshold theorem for some nonlinear integral equations. SIAM J. Math. Anal. 9 (1978), $356-$ 376.

[22] H.L. Smith, A abstract threshold theorem for one parameter families of positive noncompact operators. Funkcial. Ekvac. 24 (1981), 141-153.

[23] A.C. Thompson, On certain contraction mappings in a partially ordered vector space. Proc. Amer. Math. Soc. 14 (1963), $438-$ 443.

[24] R. Torrejón, Positive almost periodic solutions of a state dependent delay nonlinear integral equation. Nonlinear Anal. 20 (1993), 1383-1416.

[25] T. Yoshizawa, Stability Theory and the Existence of Periodic Solutions and Almost Periodic Solutions, New York, 1975.

[26] C. Zhang, Integration of vector valued pseudo almost periodic functions. Proc. Amer. Math. Soc. 121 (1994), 167-174.

[27] C. Zhang, Pseudo almost periodic functions of some differential equations. J. Math. Anal. Appl. 181 (1994), 62-676.

[28] C. Zhang, Pseudo almost periodic functions of some differential equations II. J. Math. Anal. Appl. 192 (1995), 543-561. 